\title{
Semi-algebraic description of the equilibria of dynamical systems
}

\author{
Changbo Chen, Marc Moreno Maza \\ The University of Western Ontario, London N6A 1M8, Canada
}

\begin{abstract}
We study continuous dynamical systems defined by autonomous ordinary differential equations, themselves given by parametric rational functions. For such systems, we provide semi-algebraic descriptions of their hyperbolic and non-hyperbolic equilibria, their asymptotically stable hyperbolic equilibria, their Hopf bifurcations. To this end, we revisit various criteria on sign conditions for the roots of a real parametric univariate polynomial. In addition, we introduce the notion of comprehensive triangular decomposition of a semi-algebraic system and demonstrate that it is well adapted for our study.
\end{abstract}

\section{Introduction}

The study of polynomial dynamical systems by means of symbolic computation is one of the most popular application of computer algebra. Equilibria, limit cycles, center manifolds, normal forms and bifurcation analysis are the main notions used in the study of dynamical systems $[30,4,21,33]$. These objects can be manipulated by a variety of symbolic methods $[11,9,10,39,13,34,27,23$, $24,35,19,17,16,22,36,5,31]$. Among these notions, those which have received the greatest attention by the computer algebra community are equilibria and bifurcation analysis. Studying them for polynomial dynamical systems typically consists of: (1) setting up a (parametric) semi-algebraic system $\mathcal{S},(2)$ extracting from $\mathcal{S}$ a particular information.

The aim of this paper is twofold. Our first objective is to revisit the results that are practically useful for conducting equilibrium and bifurcation by means of symbolic computation. These results are gathered in Sections 2 and 3. They are generally stated in terms of the coefficients of a univariate polynomial and translate into semi-algebraic systems. A prototype of such results is the RouthHurwitz's criterion. While many of these criteria appear in the literature (for instance in $[23,24]$ ) we also provide some new criteria, like Theorem 9 , as well as new interpretation of classical results, like Theorem 13.

Our second objective is to exhibit tools that are well adapted for solving the semi-algebraic systems implementing the above mentioned results. Typical problems on parametric dynamical systems (see Problems 1, 2, 3) require to decompose the parameter space into connected semi-algebraic sets above which the qualitative behavior of the dynamical system is essentially constant. Taking also into consideration the fact that certain degenerated behaviors have no practical 
interest, we introduce, in Section 4, the notion of a comprehensive triangular decomposition of a parametric semi-algebraic system, together with an algorithm for computing it. This work extends some of our previous papers $[6,8]$.

This paper attempts to be as self-contained and comprehensive as possible. While this is not a survey paper (as it contains new theorems and algorithms) a fair amount of classical results are recalled for the reader's convenience. In addition, we provide in Appendix A a complete process for analyzing the bistability of a biological model with the tools presented in this paper.

We dedicate the rest of this introduction to identify problems arising in the study of dynamical systems which are eligible to solutions based on semialgebraic system solving. Some of these problems, namely Problems 1, 2, 3, are directly formulated in terms of dynamical systems. For a sake of clarity, the other problems, namely Problems 4 and 5, are stated in terms of conditions on the roots of a parametric univariate polynomial, which is meant to be the characteristic polynomial of the Jacobian matrix of the dynamical system under study.

We consider continuous dynamical systems defined by autonomous ordinary differential system of the following shape:

$$
\left\{\begin{aligned}
\dot{y_{1}}= & F_{1}\left(u_{1}, \ldots, u_{d}, y_{1}, \ldots, y_{m}\right), \\
\dot{y_{2}}= & F_{2}\left(u_{1}, \ldots, u_{d}, y_{1}, \ldots, y_{m}\right), \\
\vdots & \vdots \\
\dot{y_{m}}= & F_{m}\left(u_{1}, \ldots, u_{d}, y_{1}, \ldots, y_{m}\right) .
\end{aligned}\right.
$$

where $F_{1}, \ldots, F_{m}$ are polynomials of $\mathbb{Q}\left[u_{1}, \ldots, u_{d}, y_{1}, \ldots, y_{m}\right]$. The variables $\mathbf{u}=$ $\left(u_{1}, \ldots, u_{d}\right)$ are considered as parameters and the variables $\mathbf{y}=\left(y_{1}, \ldots, y_{m}\right)$ are seen as unknowns. In addition, we have $y_{i}=y_{i}(t)$ and $\dot{y}_{i}=\mathrm{d} y_{i} / \mathrm{d} t$ while the parameters $u_{1}, \ldots, u_{d}$ are independent of the derivation variable $t$. In the sequel, we simply write (1) as

$$
\dot{\mathbf{y}}=F(\mathbf{u}, \mathbf{y})
$$

where $F(\mathbf{u}, \mathbf{y})=\left(F_{1}(\mathbf{u}, \mathbf{y}), \ldots, F_{m}(\mathbf{u}, \mathbf{y})\right)$ is called the vector field of the system.

For any given parameter value $u \in \mathbb{R}^{d}$, one may notice that any $y \in \mathbb{R}^{m}$ such that $F_{1}(u, y)=\cdots=F_{m}(u, y)=0$ holds, is a constant solution of System (1), which is called an equilibrium (or a steady state, or a fixed point). We are interested in the following problem regarding the equilibria of the given dynamical system.

Problem 1 For a fixed parameter value u (or in absence of parameters) determine the number of equilibria of (1) and compute each of them (for instance, by means of isolation intervals). In presence of parameters, partition the parameter space into connected semi-algebraic sets, such that above each of them, the number of equilibria is constant and each equilibrium is a continuous function of the parameters.

Problems 1 is a particular instance of the solving of semi-algebraic systems. Section 4 is dedicated to this more general question, with a view toward Problem 1. 
We consider now a fixed parameter value $u$ and a particular equilibrium $y$ of System (1) at $u$. An important problem concerning the equilibrium $y$ is to analyze its stability. We say $y$ is stable if any solution of System (1) starting out close to $y$ remains close to it. We say $y$ is asymptotically stable if $y$ is stable and if the solutions of System (1) starting out close to $y$ become arbitrary close to it. If $y$ is not stable, it is said to be unstable. The above discussion leads to enhance Problem 1 into the following ones, which deals with the number of asymptotically stable equilibria of System (1) depending or not on parameters.

Problem 2 For a fixed parameter value u (or in absence of parameters) determine the number of asymptotically stable hyperbolic equilibria of (1) and compute each of them. In presence of parameters, partition the parameter space into connected semi-algebraic sets, such that above each of them, the number of asymptotically stable hyperbolic equilibria is constant and each of these equilibria is a continuous function of the parameters.

The study of the system near the particular equilibrium $y$ is usually done using the linear system

$$
\dot{\mathbf{y}}=J(u, y)(\mathbf{y}-y),
$$

where $J$ is the Jacobian matrix of $F$ :

$$
J=\left(\begin{array}{cccc}
\frac{\partial F_{1}}{\partial y_{1}} & \frac{\partial F_{1}}{\partial y_{2}} & \cdots & \frac{\partial F_{1}}{\partial y_{m}} \\
\frac{\partial F_{2}}{\partial y_{1}} & \frac{\partial F_{2}}{\partial y_{2}} & \cdots & \frac{\partial F_{2}}{\partial y_{m}} \\
\vdots & \vdots & & \vdots \\
\frac{\partial F_{m}}{\partial y_{1}} & \frac{\partial F_{m}}{\partial y_{2}} & \cdots & \frac{\partial F_{m}}{\partial y_{m}}
\end{array}\right)
$$

We denote by

$$
f(\lambda)=a_{0} \lambda^{m}+a_{1} \lambda^{m-1}+a_{2} \lambda^{m-2}+\cdots+a_{m-1} \lambda+a_{m},
$$

where $a_{0}=1$, the characteristic polynomial of $J$. If the matrix $J(u, y)$ has no eigenvalues with zero real parts, that is, if $f(u, y, \lambda)$ has no roots with zero real parts, then $y$ is called a hyperbolic equilibrium at $u$; otherwise $y$ is a nonhyperbolic equilibrium at $u$. In [32], Hartman and Grobman proved the following result: if $y$ is a hyperbolic equilibrium, then near $y$, the phase portrait of the dynamical system (1) is topologically equivalent to that of the linearized dynamical system (3). The results imply that, for a hyperbolic equilibrium $y$, the phase flow of (1) is asymptotically stable near $y$ if and only if the phase flow of (3) is asymptotically stable near $y$. Therefore, using standard results on linear differential systems [1], the phase flow of (1) is asymptotically stable near $y$ if and only if all the complex roots of $f(u, y, \lambda)$ have negative real parts. This reduces Problem 2 to the following problem.

Problem 2' For a univariate polynomial $f(x) \in \mathbb{R}[x]$, determine whether all the complex roots of $f(x)$ have negative real parts or not.

In the above analysis, we assume the equilibrium $y$ is hyperbolic, so a natural question is how to determine whether $y$ is hyperbolic or not. In other words, we want to solve the following problem: 
Problem 3 For a fixed parameter value u, determine whether each equilibrium of (1) is hyperbolic or not. In presence of parameters, partition the parameter space into connected semi-algebraic sets, such that above each of them, an equilibrium is always either hyperbolic or non-hyperbolic.

This problem is equivalent to determine whether all the complex roots of the characteristic polynomial $f(u, y, \lambda)$ have nonzero real parts, which leads to the following general problem.

Problem 3' For a univariate polynomial $f(x) \in \mathbb{R}[x]$, determine whether $f(x)$ has complex roots with zero real parts or not.

When $y$ is a non-hyperbolic equilibrium of (1), if the characteristic polynomial $f(u, y, \lambda)$ has at least one complex root with positive real part, then $y$ is an unstable equilibrium. Otherwise, the stability of $y$ depends also on the higher order terms of the Taylor expansion of $F$ near the point $y$. In this situation, one usually needs to apply the Centre Manifold Theorem [3] to reduce the original system to a low dimensional dynamical system defined on a centre manifold and further simplify it by computing its normal form. Finally, the normal form can be further reduced by removing terms that do not affect the stability of the equilibrium. Therefore, the first step towards stability analysis of nonhyperbolic equilibria of (1) is to determine when the characteristic polynomial has at least one complex root with positive real part or, equivalently, determine when $f(u, y, \lambda)$ has only complex roots with non-positive real parts, which leads to the following problem.

Problem 4 For a univariate polynomial $f(x)$ with parametric coefficients, determine whether $f(x)$ has at least one complex root with positive real part. Equivalently, given two integers $k_{1}$ and $k_{2}$, determine whether $f(x)$ has zero as a root of multiplicity $k_{1}$ and $k_{2}$ pairs of purely imaginary roots while all the other complex roots have negative real parts.

When non-hyperbolic equilibria are present, another more interesting phenomenon is the appearance of bifurcation. For the dynamical system (1), a $b i$ furcation occurs at a parameter $\alpha_{0}$ if there are parameter values $\alpha_{1}$ arbitrarily close to $\alpha_{0}$ with dynamics topologically non-equivalent to those at $\alpha_{0}$. For example, the number or stability of equilibria or periodic orbits of (1) may change with perturbations of $u$ from $\alpha_{0}$. For a general dynamical system, such as (1), a systematic study is difficult. However, given an equilibrium $y$ of (1) at $u$, necessary conditions for bifurcation can be obtained as follows. If a bifurcation of an equilibrium occurs near $(u, y)$, then either or both conditions below are met:

- the characteristic polynomial $f$ has zero as a root of multiplicity $k$, for some $k>0$,

- the characteristic polynomial $f$ has $k$ pairs of purely imaginary roots, for some $k>0$. 
Therefore, the last problem we want to answer in this paper is as follows:

Problem 5 Given non-negative integers $k_{1}, k_{2}$ and a polynomial $f(x)$ with parametric coefficients, determine whether $f(x)$ has zero as a root of multiplicity $k_{1}$ and $k_{2}$ pairs of purely imaginary roots while no other roots have zero real parts.

A particular case of the above problem is $\left(k_{1}, k_{2}\right)=(0,1)$. In this case, thus if the characteristic polynomial $f(u, y, \lambda)$ has a pair of purely imaginary roots and no other roots with zero real part, the limit cycle bifurcation that may occur at $(u, y)$ is called a Hopf bifurcation. Such bifurcation has attracted the interest of many authors. In [20], the authors presented sufficient conditions for the appearance of Hopf bifurcations. In [23], the authors give sufficient and necessary conditions on Hopf bifurcations by further demanding that all the other eigenvalues have negative real roots, which is convenient for applying Centre Manifold Theory in order to reduce the dimension of dynamical systems. In [24], the authors present a framework for solving Problem 5.

\section{On the complex roots of a univariate polynomial}

As we have seen in the previous section, many problems related to dynamical systems reduce to studying the complex roots of a univariate polynomial with real coefficients. In particular, Problems 2', 3', 4 and 5 will be completely answered in the present section.

This section is firmly rooted in the papers [23,24]. With respect to $[23,24]$ our main contribution in this section is Theorem 9 , from which the main result of [23] (that is, Theorem 3.6 in [23] and Corollary 3 in this section), dedicated to Hopf bifurcation, can easily be derived. Theorem 9 provides two equivalent conditions for a polynomial with real coefficients to have only complex roots with non-positive real parts.

The proof of the first condition relies on several results of $[23,24]$, which are reviewed hereafter for the reader's convenience. To prove the second condition, we introduce Corollary 2 and Theorem 7 . It should be pointed out that to deduce Corollary 3 from Theorem 9, this second condition is really needed. We also correct the error of sign difference in Theorem 3.1 of [23](Theorem 1 in [24]) and revise it as Theorem 5 hereafter.

Let $f(x) \in \mathbb{R}[x]$ be a polynomial of degree $m$, and let us write

$$
f(x)=a_{0} x^{m}+a_{1} x^{m-1}+\cdots+a_{m} .
$$

After recalling the definition and standard properties (Lemma 1, Theorems 1, $3,2,4)$ of Hurwitz determinants, we discuss their relations with subresultant sequences in Section 2.2 and their use in the study of symmetric roots in Section 2.3.

Definition 1 (Hurwitz matrix) We call Hurwitz matrix of $f$ the $m \times m m a$ trix $H=\left(H_{\mu \nu}\right)$ defined by $H_{\mu \nu}=a_{2 \nu-\mu}$ for $\nu=1, \ldots, m$ and $\mu=1, \ldots, m$, 
with the convention that $a_{i}=0$ holds as soon as $i<0$ or $i>m$ holds. For $i=1, \ldots, m$, we denote by $\Delta_{i}$ the leading principal minors of $H$, which are called the Hurwitz determinants of $H$ :

$$
\Delta_{1}=a_{1}, \Delta_{2}=\left|\begin{array}{ll}
a_{1} & a_{3} \\
a_{0} & a_{2}
\end{array}\right|, \ldots, \Delta_{m}=\left|\begin{array}{ccccc}
a_{1} & a_{3} & a_{5} & \ldots & \ldots \\
a_{0} & a_{2} & a_{4} & \ldots & \ldots \\
0 & a_{1} & a_{3} & a_{5} & \ldots \\
0 & a_{0} & a_{2} & a_{4} & \ldots \\
& & & & \ddots
\end{array}\right| .
$$

It is easy to see that we have $\Delta_{m}=a_{m} \Delta_{m-1}$.

The following criterion provides a sufficient and necessary condition for a polynomial $f$ to have only roots with negative real parts, which is therefore an answer to Problem 1.

Theorem 1 (Routh-Hurwitz's criterion [15]) The real parts of all the zeros of $f(\lambda)$ are negative if and only if $\Delta_{1}>0, \Delta_{2}>0, \ldots, \Delta_{m-1}>0, a_{m}>0$.

There is also another famous criterion equivalent to the above one, which is called Liénard-Chipart's Criterion.

Theorem 2 (Liénard-Chipart's criterion [15]) The real parts of all the zeros of $f(\lambda)$ are negative if and only if we have:

(1) If $m$ is odd, then all the below inequalities hold:

$a_{m}>0, a_{2}>0, a_{4}>0, \ldots, a_{m-1}>0, \Delta_{2}>0, \Delta_{4}>0, \ldots, \Delta_{m-1}>0$.

(2) If $m$ is even, then all the below inequalities hold:

$a_{m}>0, a_{1}>0, a_{3}>0, \ldots, a_{m-1}>0, \Delta_{1}>0, \Delta_{3}>0, \ldots, \Delta_{m-1}>0$.

\subsection{Hurwitz determinants and stability of hyperbolic equilibria of dynamical system}

In this section, for a fixed parameter value $u \in \mathbb{R}^{d}$, let $y \in \mathbb{R}^{m}$ be an equilibrium of dynamical system (1).

Lemma 1 (Orlando's formula [14]) Let $\lambda_{i}, i=1, \ldots, m$, be the eigenvalues of $J(u, y)$ and $\Delta_{m-1}$ be the $(m-1)$-th Hurwitz determinant of its characteristic polynomial. Then we have:

$$
\Delta_{m-1}=(-1)^{\frac{1}{2} m(m-1)} \prod_{1 \leq i<j \leq m}\left(\lambda_{i}+\lambda_{j}\right) .
$$

Corollary 1 (Hyperbolic equilibrium criterion) The following three properties hold. 
(1) $J(u, y)$ have no zero eigenvalues if and only if $|J(u, y)|=(-1)^{m} a_{m} \neq 0$.

(2) If $\Delta_{m-1} \neq 0$, then $J(u, y)$ has no pure imaginary eigenvalues.

(3) If $\Delta_{m}=a_{m} \Delta_{m-1} \neq 0$, then $y$ is a hyperbolic equilibrium.

Proof. Property (1) is clear. Property (2) is an immediate consequence of Orlando's Formula. Property (3) follows from $|J(u, y)|=\lambda_{1} \lambda_{2} \cdots \lambda_{m}$.

Remark 1 Necessary and sufficient conditions for $J(u, y)$ to have no pure imaginary eigenvalues (resp. y to be hyperbolic equilibrium) will be provided in Section 2.3.

Theorem 3 (Lyapunov's first method on stability [28]) The following properties hold.

(i) If $J(u, y)$ has at least one eigenvalue with positive real parts, then $y$ is unstable.

(ii) Assume that $y$ is a hyperbolic equilibrium. If all the eigenvalues of $J(u, y)$ have negative real parts, then $y$ is asymptotically stable.

Theorem 4 (Stability criterion for hyperbolic equilibria) Let y be an equilibrium of System (1), we have:

(1) $y$ is an asymptotically stable hyperbolic equilibrium if and only if

$$
\Delta_{1}>0, \Delta_{2}>0, \ldots, \Delta_{m-1}>0, a_{m}>0 .
$$

(2) If $y$ is hyperbolic, then $y$ is unstable if and only if there exists some $i, 1 \leq$ $i \leq n$, such that $\Delta_{i} \leq 0$.

Proof. Directly by Theorem 3 and Routh-Hurwitz Criterion.

\subsection{Hurwitz determinants and subresultant sequences}

Let $\mathbb{A}$ be a commutative ring with identity and let $p \leq q$ be two positive integers. Let $M$ be a $p \times q$ matrix with coefficients in $\mathbb{A}$. Let $M_{i}$ be the square submatrix of $M$ consisting of the first $p-1$ columns of $M$ and the $i_{\text {th }}$ column of $M$, for $i=p \cdots q$. Let $\operatorname{det} M_{i}$ be the determinant of $M_{i}$. We denote by $\operatorname{dpol}(M)$ the element of $\mathbb{A}[y]$, called the determinant polynomial of $M$, given by

$$
\operatorname{det} M_{p} y^{q-p}+\operatorname{det} M_{p+1} y^{q-p-1}+\cdots+\operatorname{det} M_{q} .
$$

Let $f_{1}(y), \ldots, f_{p}(y)$ be a set of polynomials of $\mathbb{A}[y]$. Let

$$
q=1+\max \left(\operatorname{deg} f_{1}(y), \ldots, \operatorname{deg} f_{p}(y)\right) .
$$

The matrix $M$ of $f_{1}, \ldots, f_{p}$ is defined by $M_{i j}=\operatorname{coeff}\left(f_{i}, y^{q-j}\right)$.

Let $f=a_{m} y^{m}+\cdots+a_{0}, g=b_{n} y^{n}+\cdots+b_{0}$ be two polynomials of $\mathbb{A}[y]$ with positive degrees $m$ and $n$. Let $\lambda=\min (m, n)$. Denote by $\operatorname{lc}(f)$ and $\operatorname{lc}(g)$ respectively the leading coefficient of $f$ and $g$ w.r.t. $y$. For any $0 \leq i<\lambda$, let 
$M$ be the matrix of the polynomials $y^{n-1-i} f, \ldots, y f, f, y^{m-1-i} g, \ldots, y g, g$. We define the $i_{\text {th }}$ subresultant of $f$ and $g$, denoted by $S_{i}(f, g, y)$ as

$$
\begin{aligned}
S_{i}(f, g, y) & =\operatorname{dpol}\left(y^{n-1-i} f, \ldots, y f, f, y^{m-1-i} g, \ldots, y g, g\right) \\
& =\operatorname{dpol}(M) .
\end{aligned}
$$

Note that $S_{i}(f, g, y)$ is a polynomial in $\mathbb{A}[y]$ with degree at most $i$. Let $s_{i}(f, g, y)=$ $\operatorname{coeff}\left(S_{i}(f, g, y), y^{i}\right)$ and call it the principle subresultant coefficient of $S_{i}(f, g, y)$. If $m \geq n$, we define $S_{\lambda}(f, g, y)=g, S_{\lambda+1}(f, g, y)=f, s_{\lambda}=\operatorname{lc}(g)$ and $s_{\lambda+1}=$ $\operatorname{lc}(f)$. If $m<n$, we define $S_{\lambda}=f, S_{\lambda+1}=g, s_{\lambda}=\operatorname{lc}(f)$ and $s_{\lambda+1}=\operatorname{lc}(g)$.

Let $\mathbb{A}=\mathbb{Q}\left[a_{0}, \ldots, a_{m}\right]$ and $f \in \mathbb{A}[x]=a_{0} x^{m}+a_{1} x^{m-1}+\cdots+a_{m-1} x+a_{m}$ be a polynomial of degree $m$. We write $f(x)=f_{1}\left(x^{2}\right)+x f_{2}\left(x^{2}\right)$. If $m=2 \ell+1$, we have $f_{1}(y)=a_{1} y^{\ell}+a_{3} y^{\ell-1}+\cdots+a_{2 \ell+1}$ and $f_{2}(y)=a_{0} y^{\ell}+a_{2} y^{\ell-1}+\cdots+a_{2 \ell}$. If $m=2 \ell$, we have $f_{1}(y)=a_{0} y^{\ell}+a_{2} y^{\ell-1}+\cdots+a_{2 \ell}$ and $f_{2}(y)=a_{1} y^{\ell-1}+a_{3} y^{\ell-2}+\cdots+a_{2 \ell-1}$.

Theorem 5 Let $\Delta_{1}, \Delta_{2}, \ldots, \Delta_{m}$ be the Hurwitz determinants sequence of $f$. Then the following conclusion holds:

(i) If $m=2 \ell+1$, we have $\Delta_{m-1-2 i}=\Delta_{2 \ell-2 i}=(-1)^{\frac{(\ell-i)(\ell-i-1)}{2}} s_{i}\left(f_{1}, \ell, f_{2}, \ell, y\right)$ hold, for $i=0,1, \ldots, \ell-1$.

(ii) If $m=2 \ell$, we have $\Delta_{m-1-2 i}=\Delta_{2 \ell-1-2 i}=(-1)^{\frac{(\ell-i)(\ell-i-1)}{2}} s_{i}\left(f_{1}, \ell, f_{2}, \ell-\right.$ $1, y)$, for $i=0,1, \ldots, \ell-1$.

(iii) If $m=2 \ell+1$, for $i=0,1, \ldots, \ell$, we have

$$
\begin{aligned}
\Delta_{m-2 i}=\Delta_{2 \ell+1-2 i} & =(-1)^{\frac{(\ell-i)(\ell-i+1)}{2}} s_{i}\left(f_{1}, \ell, y f_{2}, \ell+1, y\right) \\
& =(-1)^{\frac{3(\ell-i)(\ell-i+1)}{2}} s_{i}\left(y f_{2}, \ell+1, f_{1}, \ell, y\right) .
\end{aligned}
$$

(iv) If $m=2 \ell$, we have $\Delta_{m-2 i}=\Delta_{2 \ell-2 i}=(-1)^{\frac{(\ell-i)(\ell-i+1)}{2}} s_{i}\left(f_{1}, \ell, y f_{2}, \ell, y\right)$ hold, for $i=0,1, \ldots, \ell-1$.

Proof. Here, we only prove $(i)$ holds and leave the other cases for exercise.

When $m=2 \ell+1$, we have $f_{1}(y)=a_{1} y^{\ell}+a_{3} y^{\ell-1} \cdots+a_{m}, f_{2}(y)=a_{0} y^{\ell}+$ $a_{2} y^{\ell-1} \cdots+a_{m-1}$. So the Sylvester matrix $M$ formed by the coefficients of $f_{1}$ and $f_{2}$ is an $2 \ell \times 2 \ell$ matrix of the form:

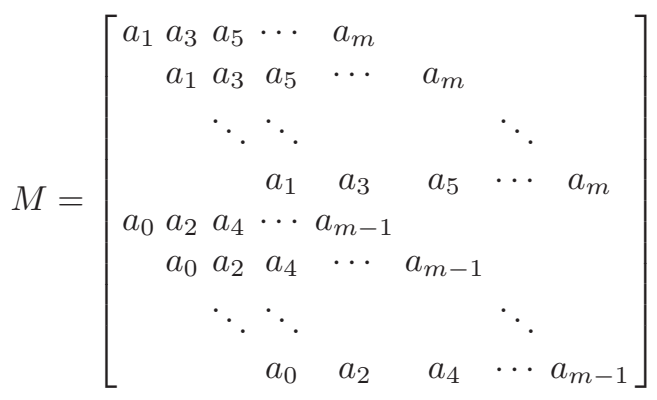


On the other hand, the Hurwitz matrix $H$ of $f$ is an $(2 \ell+1) \times(2 \ell+1)$ matrix whose elements are arranged like this:

$$
H=\left[\begin{array}{ccccccc}
a_{1} & a_{3} & a_{5} & \cdots & a_{m} & & \\
a_{0} & a_{2} & a_{4} & \cdots & a_{m-1} & & \\
& a_{1} & a_{3} & a_{5} & \cdots & a_{m} & \\
a_{0} & a_{2} & a_{4} & \cdots & a_{m-1} & \\
& & \cdots & \cdots & & \\
& a_{1} & a_{3} & a_{5} & \cdots & a_{m} \\
& a_{0} & a_{2} & a_{4} & \cdots & a_{m-1} \\
& & a_{1} & a_{3} & \cdots & a_{m-2} \\
& & & & & &
\end{array}\right]
$$

Let $H^{*}$ be the sub-matrix composed by the first $2 \ell$ rows and $2 \ell$ columns of $H$. We denote by $H_{2 i}$ the sub-matrix of $H^{*}$, formed by the first $2 i$ rows and $2 i$ columns, for $i=1,2, \ldots, \ell$. We denote by $M_{i}$ the sub-matrix of $M$, formed by deleting the last $i$ rows composed by the coefficients of $f_{1}(y)$ and the last $i$ rows composed by the coefficients of $f_{2}(y)$ and then deleting the last $2 i$ columns for $i=0,1, \ldots, \ell-1$. Then it's easy to see that if we make the odd rows of $H_{2 \ell-2 i}$ "float up" one by one, we finally get the matrix $M_{i}$. So the number of row exchanges for $H_{2 \ell-2 i}$ is: $0+1+2+\cdots+(\ell-i-1)=\frac{(\ell-i)(\ell-i-1)}{2}$. Therefore, we have $\Delta_{2 \ell-2 i}=\left|H_{2 \ell-2 i}\right|=(-1)^{\frac{(\ell-i)(\ell-i-1)}{2}}\left|M_{i}\right|=(-1)^{\frac{(\ell-i)(\ell-i-1)}{2}} s_{i}\left(f_{1}, \ell, f_{2}, \ell, y\right)$, for $i=0,1, \ldots, \ell-1$.

Remark 2 This theorem is a corrected version of Theorem 1 in [24], where the sign differences between $\Delta_{i}$ and $s_{i}$ are wrong.

\subsection{Hurwitz determinants and symmetric roots}

The following result is taken from [23]. Corollary 2 is a direct consequence.

Lemma 2 ([23]) Given a univariate polynomial $f(x)=a_{0} x^{m}+a_{1} x^{m-1}+\cdots+$ $a_{m}$ of $\mathbb{R}[x]$, where $a_{0} \neq 0$. We write $f(x)$ into the form: $f(x)=f_{1}\left(x^{2}\right)+x f_{2}\left(x^{2}\right)$. Then $f(x)$ has a pair of symmetric zeros $z$ and $-z$ in $\mathbb{C}$ if and only if $z^{2}$ is a common zero of $f_{1}(y)$ and $f_{2}(y)$.

Corollary 2 Assume that $a_{m} \neq 0$, then $f(x)$ has a pair of symmetric zeros $z$ and $-z$ in $\mathbb{C}$ if and only if $z^{2}$ is a common zero of $f_{1}(y)$ and $y f_{2}(y)$.

Theorem 6 ([23]) Let $f(x)=a_{0} x^{m}+a_{1} x^{m-1}+\cdots+a_{m} \in \mathbb{R}[x]$ be a polynomial of degree $m$. Then $f(x)$ has exactly $k$ pairs of symmetric roots $z_{i}$ and $-z_{i}$ in $\mathbb{C}$ if and only if $\Delta_{m-1}=0, \ldots, \Delta_{m-2 k+1}=0, \Delta_{m-2 k-1} \neq 0$.

Theorem 7 Notation as above, if $a_{m} \neq 0$, then $f$ has exactly $k$ pairs of symmetric roots $z_{i}$ and $-z_{i}$ if and only if $\Delta_{m}=0, \ldots, \Delta_{m-2 k+2}=0, \Delta_{m-2 k} \neq 0$.

Proof. If $a_{m} \neq 0$, by Corollary 2 , the number of symmetric roots, counted with multiplicities, of the polynomial $f$ is equal to the number of common roots, 
counted with multiplicities, of the two polynomials $f_{1}(y)$ and $y f_{2}(y)$. According to the elementary properties of subresultant sequences the polynomials $f_{1}(y)$ and $f_{2}(y)$ have $k$ common roots if and only if

$$
s_{0}\left(f_{1}, y f_{2}, y\right)=0, \ldots, s_{k-1}\left(f_{1}, y f_{2}, y\right)=0, s_{k}\left(f_{1}, y f_{2}, y\right) \neq 0 .
$$

So by Theorem 5 and specialization property of subresultants $[29,7], f$ has exactly $k$ pairs of symmetric roots if and only if $\Delta_{m}=0, \ldots, \Delta_{m-2 k+2}=$ $0, \Delta_{m-2 k} \neq 0$.

Lemma 3 ([23]) Let $f(x) \in \mathbb{R}[x]$ be a polynomial of degree $m$ and $z_{1}, \ldots, z_{k}$ be arbitrary complex numbers. Let $f^{*}(x)=f(x)\left(x^{2}-z_{1}^{2}\right) \cdots\left(x^{2}-z_{k}^{2}\right)$. If $\Delta_{i}^{*}$ is the Hurwitz determinants of order $i$ of the polynomial $f^{*}(x)$, then $\Delta_{i}=\Delta_{i}^{*}$, for $i=1, \ldots, m$. Similarly, let $f^{*}(x)=f(x) x^{k}$, then we also have $\Delta_{i}=\Delta_{i}^{*}$ hold.

Theorem 8 The polynomial $f(x)$ has zero as root of multiplicity $k$ and all the other roots in the left half-plane if and only if $a_{m-k+1}=\cdots=a_{m}=0$ and $\Delta_{1}>0, \Delta_{2}>0, \ldots, \Delta_{m-k}>0$.

Proof. It follows directly from Routh-Hurwitz criterion and Lemma 3.

Theorem 9 Let $f(x) \in \mathbb{R}[x]$ be a polynomial of degree $m$ and $f(x)=a_{0} x^{m}+$ $a_{1} x^{m-1}+\cdots+a_{m}=f_{1}\left(x^{2}\right)+x f_{2}\left(x^{2}\right)$. Let $\Delta_{1}, \Delta_{2}, \ldots, \Delta_{m}$ be the Hurwitz determinants sequence of $f$. Then the following statements are equivalent:

(i) $f(x)$ has $k$ pairs of pure imaginary roots and all the other roots are in the left half-plane.

(ii) $S_{k}\left(f_{1}, f_{2}, y\right)$ has $k$ negative real roots and $\Delta_{m-1}=\Delta_{m-3}=\cdots=\Delta_{m-2 k+1}=$ $0, \Delta_{m-2 k}>0, \Delta_{m-2 k-1}>0, \ldots, \Delta_{1}>0$.

(iii) $S_{k}\left(f_{1}, y f_{2}, y\right)$ has $k$ negative real roots and $a_{m} \neq 0, \Delta_{m}=\Delta_{m-2}=\cdots=$ $\Delta_{m-2 k+2}=0, \Delta_{m-2 k}>0, \Delta_{m-2 k-1}>0, \ldots, \Delta_{1}>0$.

Proof. " $(i) \Rightarrow(i i)$ ". Assume that $f(x)$ has $k$ pairs of pure imaginary roots and all the other roots are in the left half-plane. Let $\pm i \omega_{1}, \ldots, \pm i \omega_{k}$ be the $k$ pairs of pure imaginary roots, then we can write $f(x)$ as $f(x)=f^{*}(x)\left(x^{2}+\omega_{1}^{2}\right) \cdots\left(x^{2}+\right.$ $\left.\omega_{k}^{2}\right)$, where $\omega_{1}^{2}>0, \ldots, \omega_{k}^{2}>0$ and $f^{*}(x)$ has only roots in the left half-plane. By Routh-Hurwitz criterion, we know that $\Delta_{1}^{*}>0, \Delta_{2}^{*}>0, \ldots, \Delta_{m-2 k}^{*}>0$. According to the Lemma 3 , we know that $\Delta_{i}^{*}=\Delta_{i}$. Therefore, we have $\Delta_{m-2 k}>$ $0, \Delta_{m-2 k-1}>0, \ldots, \Delta_{1}>0$ hold.

Moreover, by assumption we know the $k$ pairs of pure imaginary roots are the only symmetric roots of $f(x)$, which implies $\Delta_{m-1}=\Delta_{m-3}=\cdots=$ $\Delta_{m-2 k+1}=0, \Delta_{m-2 k-1} \neq 0$. Therefore, by Theorem 5 we have $s_{0}\left(f_{1}, f_{2}, y\right)=$ $0, \ldots, s_{k-1}\left(f_{1}, f_{2}, y\right)=0, s_{k}\left(f_{1}, f_{2}, y\right) \neq 0$, which implies that $S_{k}\left(f_{1}, f_{2}, y\right)=$ $\operatorname{gcd}\left(f_{1}, f_{2}, y\right)$. On the other hand, since $\pm i \omega_{1}, \ldots, \pm i \omega_{k}$ are the symmetric roots of $f(x)$, by Lemma $2,-\omega_{1}^{2}, \ldots,-\omega_{k}^{2}$ are the common roots of $f_{1}(y)$ and $f_{2}(y)$, that is, they are the real roots of $S_{k}\left(f_{1}, f_{2}, y\right)$. Therefore $S_{k}\left(f_{1}, f_{2}, y\right)$ has $k$ negative real roots. 
"(ii) $\Rightarrow($ i $)$ " By the assumption, we have $\Delta_{m-1}=\Delta_{m-3}=\cdots=\Delta_{m-2 k+1}=$ $0, \Delta_{m-2 k-1} \neq 0$, which implies that

$$
s_{0}\left(f_{1}, f_{2}, y\right)=s_{1}\left(f_{1}, f_{2}, y\right)=\cdots=s_{k-1}\left(f_{1}, f_{2}, y\right)=0, s_{k}\left(f_{1}, f_{2}, y\right) \neq 0 .
$$

Therefore the degree of $S_{k}\left(f_{1}, f_{2}, y\right)$ is $k$ and $S_{k}\left(f_{1}, f_{2}, y\right)=\operatorname{gcd}\left(f_{1}, f_{2}, y\right)$. Since $S_{k}\left(f_{1}, f_{2}, y\right)$ has $k$ negative real roots, we know that $f_{1}(y)$ and $f_{2}(y)$ has $k$ common negative real roots and no other common roots. So by Lemma $2, f(x)$ has exactly $k$ pairs of pure imaginary roots and no other symmetric roots. Let us write $f(x)=f^{*}(x)\left(x^{2}+\omega_{1}^{2}\right) \cdots\left(x^{2}+\omega_{k}^{2}\right)$, according to $\Delta_{m-2 k}>0, \Delta_{m-2 k-1}>$ $0, \ldots, \Delta_{1}>0$ and Lemma 3 , we know that all the roots of $f^{*}(x)$ are in the left half-plane. Therefore $f(x)$ has $k$ pairs of pure imaginary eigenvalues and all the other roots are in the left half-plane.

The proof of equivalence of $(i)$ and (iii) are similar. The only difference is that during the proof we need to use Theorem 7 instead of Theorem 6 and Corollary 2 instead of Lemma 2.

By the above theorem, we get the following corollary, which is the main theorem on Hopf bifurcation in [23, 24].

Corollary 3 (Theorem $4[\mathbf{2 4}]$ ) Let $f(x) \in \mathbb{R}[x]$ be a degree $m$ polynomial and write $f(x)=a_{0} x^{m}+a_{1} x^{m-1}+\cdots+a_{m}=f_{1}\left(x^{2}\right)+x f_{2}\left(x^{2}\right)$ with $a_{0}>0$. Let $\Delta_{1}, \Delta_{2}, \ldots, \Delta_{m}$ be the Hurwitz determinants sequence of $f$. Then $f(x)$ has a pair of distinct roots, $i \omega$ and $-i \omega$, on the imaginary and all the other roots in the left half-plane if and only if $a_{m}>0, \Delta_{m-1}=0, \Delta_{m-2}>0, \ldots, \Delta_{1}>0$.

Proof. By the equivalence of $(i)$ and (iii) in Theorem 9, we only need to prove that $a_{m}>0, \Delta_{m-1}=0, \Delta_{m-2}>0, \ldots, \Delta_{1}>0$ if and only if $S_{1}\left(f_{1}, y f_{2}, y\right)$ has one negative real root and $a_{m} \neq 0, \Delta_{m}=0, \Delta_{m-2}>0, \ldots, \Delta_{1}>0$. By Theorem 5, we have $S_{1}\left(f_{1}, y f_{2}, y\right)=(-1)^{\frac{\ell(\ell-1)}{2}}\left(\Delta_{m-2} y+a_{m} \Delta_{m-3}\right)$.

" $\Rightarrow$ " Since $a_{m}>0, \Delta_{m-1}=0$, we have $a_{m} \neq 0$ and $\Delta_{m}=a_{m} \Delta_{m-1}=0$. Moreover, as $a_{m}>0$ and $\Delta_{m-2}>0, \Delta_{m-3}>0$, we know that $S_{1}\left(f_{1}, y f_{2}, y\right)$ has one negative real root.

" $\Leftarrow$ " Since $S_{1}\left(f_{1}, y f_{2}, y\right)$ has one negative real root and $\Delta_{m-2}>0, \Delta_{m-3}>$ 0 , we have $-\Delta_{m-2} a_{m} \Delta_{m-3}<0$, which implies that $a_{m}>0$. Moreover, by $\Delta_{m}=0$, we have $\Delta_{m-1}=0$.

Combining the result of Theorem 8 and Theorem 9, we get the answer to Problem 4. The answer to Problem 5 was first briefly mentioned in [24], which we summarize as the following Theorem.

Theorem 10 Let $f(x)=a_{0} x^{m}+a_{1} x^{m-1}+\cdots+a_{m}$ be a univariate polynomial of $\mathbb{R}[x]$. Then $f(x)$ has a root 0 of multiplicity $k_{1}$ and has $k_{2}$ pairs of pure imaginary roots while no other roots have zero real parts if and only if the following holds:

- The coefficients of $f(x)$ satisfy $a_{m}=\cdots=a_{m-k_{1}+1}=0, a_{m-k_{1}} \neq 0$. 
- Denote $a_{0} x^{m-k_{1}}+a_{1} x^{m-k_{1}-1}+\cdots+a_{m-k_{1}}=f_{1}\left(x^{2}\right)+x f_{2}\left(x^{2}\right)$. Then there exists an integer $k \geq k_{2}$ such that $S_{k}\left(f_{1}, f_{2}, y\right)$ has $k_{2}$ negative real roots and

$$
\Delta_{m-k_{1}-1}=\Delta_{m-k_{1}-3}=\cdots=\Delta_{m-k_{1}-2 k+1}=0, \Delta_{m-k_{1}-2 k-1} \neq 0 .
$$

Proof. It directly follows from Lemma 2, Lemma 3 and Theorem 6.

Remark 3 In the above theorem, if both $k_{1}=0$ and $k_{2}=0$, then we get an answer to Problem $3^{\prime}$. If $k_{1}=0$ and $k_{2}=1$, then we get the necessary and sufficient condition on Hopf bifurcation.

The reader may notice that in [23, 24] there is also a theorem to provide sufficient and necessary conditions on Hopf bifurcation. More precisely, it is Theorem 3.5 in [23] and Theorem 3 in [24]. However, we find that (also noticed by the author) the condition provided there is only a sufficient condition.

In Theorem 10, we need to determine when a univariate polynomial $S_{k}$ of degree $k$ with parametric coefficients has $k_{2}, 0<k_{2} \leq k$, negative real zeros. This problem can be reduced to an exhaustive case discussion on the signs of polynomials whose variables are the coefficients of $S_{k}$, by Sturm-Habicht sequence [18] or negative root discriminant sequence [37].

In Theorem 9, rather we want to determine when all the complex roots of a univariate polynomial with parametric real coefficients are real and negative. In the rest of this section, we provide a relatively simple answer by virtue of Descartes criterion and discriminant sequence $[37,38]$.

Lemma 4 (Descartes criterion) Let $f(x) \in \mathbb{R}[x]$ be a polynomial of degree $n$. Let $\nu$ be the number of sign variations of its coefficients sequence. Then there exists $m \geq 0$ such that the number of positive real roots of $f(x)$ equals $\nu-2 m$.

Corollary 4 Let $f(x)=a_{0} x^{n}+\cdots+a_{n-1} x+a_{n}$ be a polynomial of degree $n$. If $f(x)$ has $n$ negative real roots, then we have $a_{i} a_{i+1}>0$ for all $0 \leq i \leq n-1$.

Proof. Since $f(x)$ has $n$ negative real roots, $f(-x)$ has $n$ positive real roots. By Descartes criterion, we have $a_{i} \neq 0$. On the other hand, since $f(x)$ has no positive real roots, we know that $a_{i}$ have the same sign. Done.

Definition 2 (Discrimination matrix) Given a polynomial with general symbolic coefficients, $f(x)=a_{0} x^{n}+a_{1} x^{n-1}+\cdots+a_{n}$, the following $2 n \times 2 n$ matrix in terms of the coefficients,

$$
\left[\begin{array}{cccccccc}
a_{0} & a_{1} & a_{2} & \cdots & a_{n} & & & \\
0 & n a_{0} & (n-1) a_{1} & \cdots & a_{n-1} & & & \\
0 & a_{0} & a_{1} & \cdots & a_{n-1} & a_{n} & & \\
0 & 0 & n a_{0} & \cdots & 2 a_{n-2} & a_{n-1} & & \\
& & & \cdots & \cdots & & & \\
& & & \cdots & \cdots & & & \\
& & & a_{0} & a_{1} & a_{2} & \cdots & a_{n} \\
& & & 0 & n a_{0} & (n-1) a_{1} & \cdots & a_{n-1}
\end{array}\right]
$$


is called the discrimination matrix of $f(x)$, and denoted by Discr $(f)$. By $d_{k}$ or $d_{k}(f)$ denote the determinant of the submatrix of Discr $(f)$, formed by the first $k$ rows and the first $k$ columns for $k=1,2, \ldots, 2 n$.

Definition 3 (Discriminant sequence) Let $D_{k}=d_{2 k}, k=1, \ldots, n$. We call the sequence $\left[D_{1}, D_{2}, \ldots, D_{n}\right]$ the discriminant sequence of $f(x)$, and denote it by DiscrList $(f)$. The last term $D_{n}$ is just the discriminant of $f$.

Definition 4 (Sign list) We call the list $\left[\operatorname{sign}\left(A_{1}\right), \operatorname{sign}\left(A_{2}\right), \ldots, \operatorname{sign}\left(A_{n}\right)\right]$ the sign list of a given sequence $A_{1}, A_{2}, \ldots, A_{n}$, where

$$
\operatorname{sign}\left(A_{i}\right)=\left\{\begin{array}{cc}
1, & A_{i}>0 \\
0, & A_{i}=0 \\
-1, & A_{i}<0
\end{array}\right.
$$

Definition 5 (Revised sign list) Given a sign list $\left[s_{1}, s_{2}, \ldots, s_{n}\right]$, we construct a new list $\left[t_{1}, t_{2}, \ldots, t_{n}\right]$ as follows: (which is called the revised sign list)

- If $\left[s_{i}, s_{i+1}, \ldots, s_{i+j}\right]$ is a section of the given list, where $s_{i} \neq 0, s_{i+1}=\cdots=$ $s_{i+j-1}=0, s_{i+j} \neq 0$, then, we replace the subsection $\left[s_{i+1}, \ldots, s_{i+j-1}\right]$ by the first $j-1$ terms of $\left[-s_{i},-s_{i}, s_{i}, s_{i},-s_{i},-s_{i}, s_{i}, s_{i}, \ldots\right]$.

- Otherwise, let $t_{k}=s_{k}$, i.e. no changes for other terms.

Theorem 11 Given a polynomial $f(x)=a_{0} x^{n}+a_{1} x^{n-1}+\cdots+a_{n}$, where $a_{0} \neq 0$ of $\mathbb{R}[x]$. If the number of sign changes of the revised sign list of $D_{1}, D_{2}, \ldots, D_{n}$ is $\nu$, the number of non-vanishing members of the revised sign list is $l$, then we have: the number of distinct real roots of $f(x)$ equals $l-2 \nu$; the number of distinct pairs of conjugate imaginary roots of $f(x)$ is $\nu$.

Example 1 Let $f=(x-1)\left(x^{2}+1\right)$, whose discriminant sequence is $[3,-4,-16]$. The sign list of it is: $[1,-1,1]$. Its revised is the same to the sign list. So the number of distinct real roots of $f$ is $3-2=1$.

Theorem 12 Let $f(x) \in \mathbb{R}[x]$ be a polynomial of degree $n$ and $\left[D_{1}, D_{2}, \ldots, D_{n}\right]$ be its discriminant sequence. Then $f(x)$ has $n$ negative real roots if and only if all its coefficients have the same nonzero sign and there exists $k, 1 \leq k \leq n$, such that $\forall i \leq k, D_{i}>0$ and for other $i$, we have $D_{i}=0$.

Proof. " $\Rightarrow$ " By Corollary 4, we know that all the coefficients of $f(x)$ have the same nonzero sign. On the other hand, since $f(x)$ has no imaginary real roots, the revised sign list of $\left[D_{1}, D_{2}, \ldots, D_{n}\right]$ has no sign changes according to Theorem 11. By the rule on constructing the revised sign list, we conclude that there exists $k, 1 \leq k \leq n$, such that $\forall i \leq k, D_{i}>0$ and for all $i>k, D_{i}=0$.

" $\Leftarrow$ " If there exists $k, 1 \leq k \leq n$, such that $\forall i \leq k, D_{i}>0$ and for other $i$, we have $D_{i}=0$. Then the revised sign list will look like this: $[1, \ldots, 1,0, \ldots, 0]$ Therefore, the number of sign changes is 0 . So $f(x)$ have no imaginary roots. Moreover, since the coefficients sequence of $f(x)$ has 0 sign variations, we know immediately that $f(x)$ has $n$ negative real roots by Descartes Criterion. 


\section{Stability of hyperbolic equilibria in view of bifurcation}

In Section 2, we discussed the stability of a hyperbolic equilibria for a fixed parameter value. In this section, we study the stability of a hyperbolic equilibria under variation of parameters.

Definition 6 ([25]) Let us consider a dynamical system that depends on parameters. The appearance of a topologically nonequivalent phase portrait under variation of parameters is called a bifurcation.

Lemma 5 ([25]) Given two hyperbolic equilibria of dynamical system (1), the phase portraits of system (1) near them are locally topologically equivalent if and only if at the two equilibria the Jacobian matrix $J$ has the same number of eigenvalues with negative (positive) real parts.

Theorem 13 (Boundary crossing theorem) Given a parameter value $\alpha_{0}$ of the dynamical system (1) and let $\beta_{0}$ be a hyperbolic equilibrium of system (1) at the parameter $\alpha_{0}$. Then there exists a continuous function $y(u)$ defined in a small neighbourhood $O\left(\alpha_{0}\right)$ of $\alpha_{0}$ satisfying $F(u, y(u))=0, y\left(\alpha_{0}\right)=\beta_{0}$. Moreover, the defining domain $O\left(\alpha_{0}\right)$ of $y(u)$ can be extended as long as $\Delta_{m}(u, y(u)) \neq 0$. In addition, inside the extended domain, there will be no bifurcation. In particular, the stability of $y(u)$ remains the same in the extended domain.

Proof. Since $\beta_{0}$ is a hyperbolic equilibrium of system (1), we have $\Delta_{m}\left(\alpha_{0}, \beta_{0}\right)=$ $(-1)^{m} \Delta_{m-1}\left(\alpha_{0}, \beta_{0}\right) \operatorname{Det}(J)\left(\alpha_{0}, \beta_{0}\right) \neq 0$. Since $\operatorname{Det}(J)\left(\alpha_{0}, \beta_{0}\right) \neq 0$, by the implicit function Theorem, we know that in a neighbourhood of $\alpha_{0}$, there is one and only one continuous function $y(u)$ defined by $F(u, y(u))=0$ such that $y\left(\alpha_{0}\right)=\beta_{0}$. Moreover, we can extend the domain of the function $y(u)$ if only $\operatorname{Det}(J)(u, y(u)) \neq 0$. On the other hand, the real parts of the eigenvalues of $J(u, y(u))$ will not become zero, which implies that the number of the eigenvalues of $J(u, y(u))$ with negative real parts and positive real parts will remain the same, respectively. By Lemma 5 , the phase portraits will remain locally topologically equivalent. Therefore, the stability will not change if only $\Delta_{n}(u, y(u)) \neq 0$.

Remark 4 In 1929, Frazer and Duncan published a paper entitled "On the Criteria for the Stability of Small Motions" [12]. In that paper, the authors presented a theorem with the same name as above one, where they pointed out that when the system passes from a region of stability to the border of stability, $\Delta_{n}$ changes from positive to zero. Here by the language of bifurcation, we see that a dynamical system will keep structurally stable if only the parameter does not cross the boundary described by $\Delta_{n}=0$.

\section{Comprehensive triangular decomposition of parametric semi-algebraic systems}

In this section, we introduce the notion of a comprehensive triangular decomposition of a parametric semi-algebraic system. Its purpose serves our needs in 
the study of parametric polynomial dynamical systems: solving the parametric semi-algebraic systems that arise from the results of Sections 2 and 3.

We start with some necessary notations. For the related concepts, the reader may refer to $[6,8,2]$. Let $\mathbf{k}$ is a field of characteristic zero and let $\mathbf{K}$ be its algebraic closure. Let $d, m, n$ be positive integers such that we have $n=d+m$ and $d, m \geq 1$. Let $\mathbf{x}=x_{1}<\cdots<x_{n}$ be ordered variables, which are divided into two groups $x_{1}<\cdots<x_{d}$ and $x_{d+1}<\cdots<x_{n}$. We rename $x_{i}$ as $u_{i}$ for $1 \leq i \leq d$ and see $\mathbf{u}=u_{1}, \ldots, u_{d}$ as parameters. We rename $x_{i}$ as $y_{i-d}$ for $d+1 \leq i \leq n$ and see $\mathbf{y}=y_{1}, \ldots, y_{m}$ as unknowns.

In this paper, we use " $Z$ " to denote the zero set of a polynomial system, involving equations and inequations, in $\mathbf{K}^{n}$ and " $Z_{\mathbb{R}}$ " to denote the zero set of a semi-algebraic system in $\mathbb{R}^{n}$. For a polynomial system $\mathcal{S}$ and point $u$, we denote by $\mathcal{S}(u)$ the specialized (or evaluated) system at $u$.

Let $p$ be a non-constant polynomial of $\mathbf{k}[\mathbf{x}]$. Denote by $\operatorname{sep}(p)$ the separant (that is the derivative of $p$ w.r.t. its main variable) of $p$. Let $T$ be a regular chain of $\mathbf{k}[\mathbf{u}, \mathbf{y}]$. Denote respectively by $\operatorname{mvar}(T), h_{T}, \operatorname{sep}(T)$ and $W(T)$ the set of main variables of $T$, the product of initials of polynomials in $T$, the product of all $\operatorname{sep}(p)$ for $p \in T$ and the quasi-component of $T$. Let $p \in \mathbf{k}[\mathbf{u}, \mathbf{y}]$. Denote by $\operatorname{res}(p, T)$ the iterated resultant of $p$ w.r.t. $T$. Denote by $\varnothing$ the empty regular chain.

In section 4.1, we introduce the concept of a disjoint squarefree comprehensive triangular decomposition (DSCTD) of a parametric constructible system cs of $\mathbf{k}[\mathbf{u}, \mathbf{y}]$, which extends the notion of a CTD of an algebraic variety $V$ of $\mathbf{k}[\mathbf{u}, \mathbf{y}]$, introduced in [6]. We also present an algorithm for computing this new type of decomposition.

In section 4.2, we introduce the concept of a comprehensive triangular decomposition of a parametric semi-algebraic system (RCTD) $\mathcal{S}$ of $\mathbf{k}[\mathbf{u}, \mathbf{y}]$. Moreover, we show that RCTD can be easily computed by combining DSCTD with our previous work on computing CAD via triangular decompositions [8].

\subsection{Disjoint squarefree comprehensive triangular decomposition}

Definition $\mathbf{7}$ Let $R:=[T, h]$ be a squarefree regular system of $\mathbf{k}[\mathbf{u}, \mathbf{y}]$. Let $u \in$ $\mathbf{K}^{d}$. We say that $R$ specializes well at $u$ if $R(u)$ is a squarefree regular system of $\mathbf{K}[\mathbf{y}]$ and $h_{T}(u) \neq 0$. Let $\mathcal{R}=\left\{R_{1}, \ldots, R_{e}\right\}$ be a finite set of regular systems of $\mathbf{k}[\mathbf{u}, \mathbf{y}]$. We say that $\mathcal{R}$ specializes disjointly well at $u$, if: $(i)$ each $R \in \mathcal{R}$ specializes well at $u$ and $(i i)$ the zero sets of $R_{i}(u)$ in $\mathbf{K}^{n}$ are pairwise disjoint.

Denote by $\pi_{\mathbf{u}}$ the canonical projection onto the parameter space. Let $\sqcup$ denote the disjoint union of two sets. Let $c s$ be a constructible set of $\mathbf{K}^{n}$. Following the results of [6], we assume that $c s$ is given as the union of the zero sets of finitely many regular systems in $\mathbf{k}[\mathbf{u}, \mathbf{y}]$. In this section, we always assume that $c s$ is represented by such a set of regular systems.

Definition 8 Let cs be a constructible set of $\mathbf{K}^{n}$. A DSCTD of cs is a pair $\left(\mathcal{C},\left(\mathcal{R}_{C}, C \in \mathcal{C}\right)\right)$, where $\mathcal{C}$ is a finite partition of $\pi_{\mathbf{u}}(c s)$ into nonempty con- 
structible sets, and, for each $C \in \mathcal{C}, \mathcal{R}_{C}$ is a finite set of regular systems of $\mathbf{k}[\mathbf{u}, \mathbf{y}]$ such that for each point $u \in C$ the following conditions hold:

(i) $\mathcal{R}_{C}$ specializes disjointly well at $u$;

(ii) we have $\operatorname{cs}(u)=\bullet_{R \in \mathcal{R}_{C}} Z(R(u))$

Let $R:=[T, h]$ be a squarefree regular system of $\mathbf{k}[\mathbf{u}, \mathbf{y}]$. Let $T_{\mathbf{u}}\left(\operatorname{resp} . T_{\mathbf{y}}\right)$ denote the set of polynomials in $T$ whose main variables belong to $\mathbf{u}$ (resp. $\mathbf{y}$ ). Define $r_{\mathbf{y}}=\operatorname{res}\left(h \cdot \operatorname{sep}\left(T_{\mathbf{y}}\right), T_{\mathbf{y}}\right)$. Let $W^{\mathbf{u}}\left(T_{\mathbf{u}}\right)$ be the quasi-component of $T_{\mathbf{u}}$ in $\mathbf{K}^{d}$.

Let $p \in \mathbf{k}[\mathbf{u}, \mathbf{y}]$. Denote by coeffs $(p, \mathbf{y})$ the set of coefficients of $p$ w.r.t. the variables $\mathbf{y}$. Let $V^{\mathbf{u}}(\operatorname{coeffs}(p, \mathbf{y}))$ be the algebraic variety of coeffs $(p, \mathbf{y})$ in $\mathbf{K}^{d}$.

Definition 9 We call defining set of the squarefree regular system $R:=[T, h]$ the set denoted by $D^{\mathbf{u}}(R)$ and defined by $D^{\mathbf{u}}(R):=W^{\mathbf{u}}\left(T_{\mathbf{u}}\right) \backslash V^{\mathbf{u}}\left(\operatorname{coeffs}\left(r_{\mathbf{y}}, \mathbf{y}\right)\right)$.

Lemma 6 Let $R:=[T, h]$ be a squarefree regular system of $\mathbf{k}[\mathbf{u}, \mathbf{y}]$. Let $u \in \mathbf{K}^{d}$. Then $R$ specializes well at $u$ if and only if $u \in D^{\mathbf{u}}(R)$.

Proof. Its proof is based on the specialization properties of subresultants and it is similar to the proof of Proposition 4 of [6].

Algorithm 14 computes a DSCTD of a constructible set. The proof of its termination and correctness is similar to that of the algorithm CTD in [6]. We also refer to [6] for the specifications of the subroutines MPD, SMPD and Intersect called in Algorithm 14.

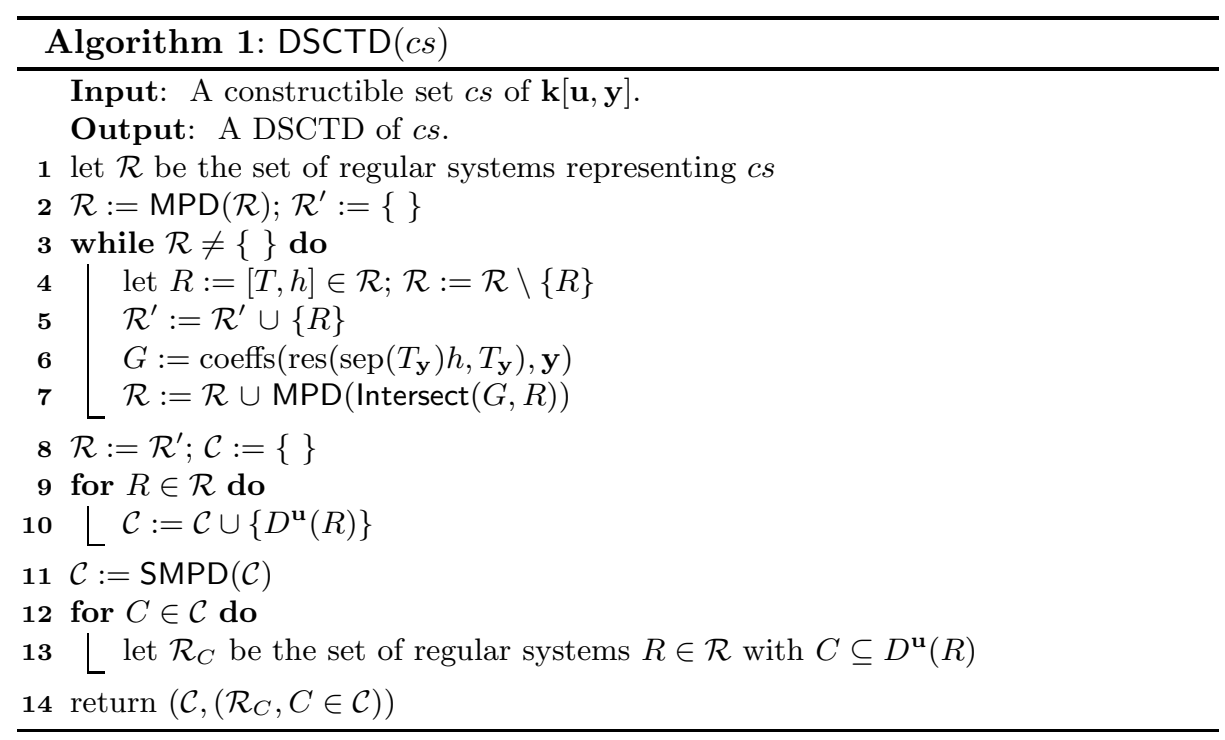


The implementation of the DSCTD algorithm is available in the RegularChains library since Maple13. It sits inside the ParametricSystemTool module and is implemented as the command ComprehensiveTriangularize with option the 'disjoint'='yes'.

Let $c s$ be a constructible set of $\mathbf{K}^{n}$. Often, we only need to partition the parameter space into constructible sets such that above each of them:

1. either $c s$ has no solutions;

2. or $c s$ has infinitely many solutions;

3. or $c s$ has a constant number of solutions and such that the solutions are continuous functions of the parameters.

A precise definition of this idea is stated in Definition 10.

Definition 10 Let cs be a constructible set of $\mathbf{K}^{n}$. A weak DSCTD (WDSCTD) of cs is a pair $\left(\mathcal{C},\left(\mathcal{T}_{C}, C \in \mathcal{C}\right)\right)$, where

$-\mathcal{C}$ is a finite partition of $\mathbf{K}^{d}$ into nonempty constructible sets,

- for each $C \in \mathcal{C}, \mathcal{T}_{C}$ is a finite set of regular chains of $\mathbf{k}[\mathbf{u}, \mathbf{y}]$ such that:

(i) either $\mathcal{T}_{C}$ is empty, which means that $c s(u)$ is empty for each $u \in C$

(ii) or $\mathcal{T}_{C}=\{\varnothing\}$, which means that cs $(u)$ is infinite for each $u \in C$;

(iii) or each $T \in \mathcal{T}_{C}$ satisfies $\operatorname{mvar}(T)=\mathbf{y}$ and for each $u \in C, \mathcal{T}_{C}$ specializes disjointly well at $u$ and $c s(u)=\cup_{T \in \mathcal{T}_{C}} Z(T(u))$.

Algorithm 2 computes a WDSCTD of $c s$. It is not difficult to prove the termination and correctness of this algorithm.

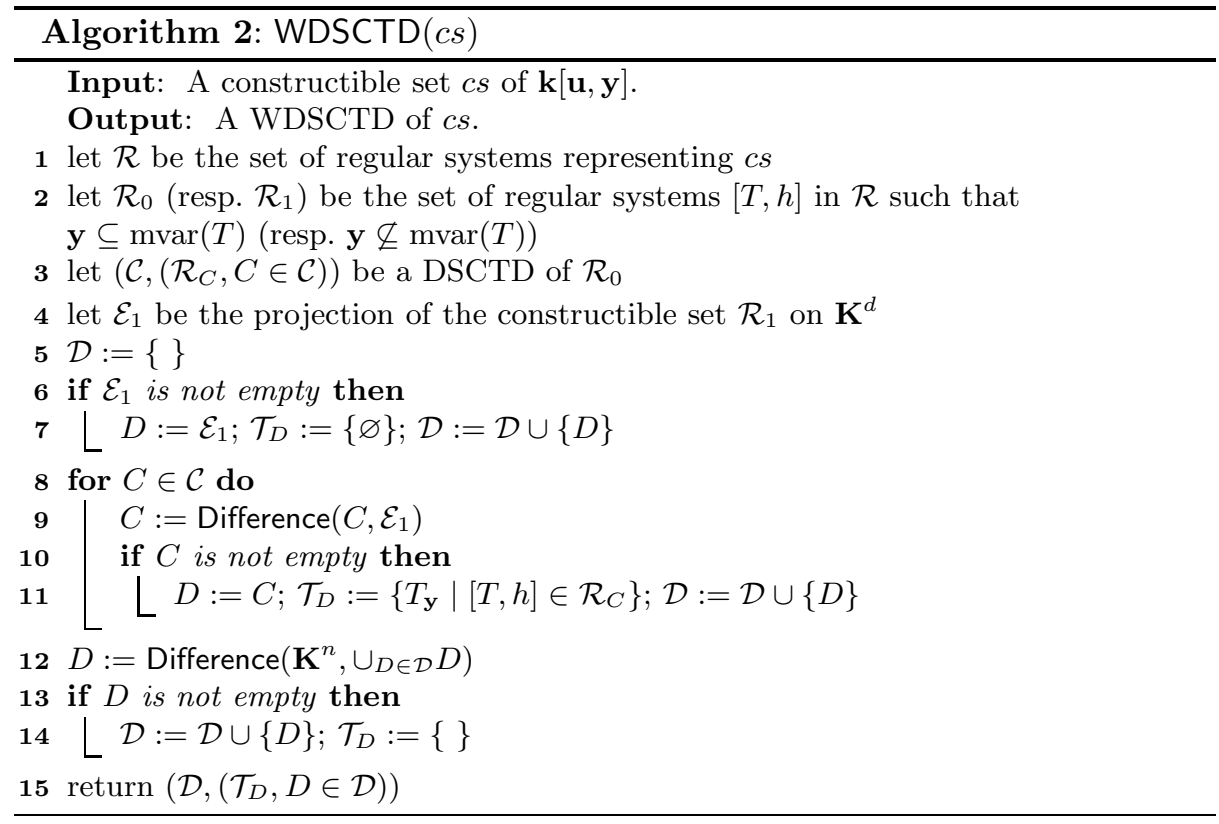




\subsection{Comprehensive triangular decomposition of a parametric semi-algebraic system}

Let $F=\left\{f_{1}, \ldots, f_{s}\right\}, P=\left\{p_{1}, \ldots, p_{r}\right\}$ be two finite sets of polynomials of $\mathbb{Q}[\mathbf{u}, \mathbf{y}]$. We denote by $\left[F, P_{>}\right]$the basic semi-algebraic system $\left\{f_{1}=0, \ldots, f_{s}=\right.$ $\left.0, p_{1}>0, \ldots, p_{r}>0\right\}$. Its zero set in $\mathbb{R}^{n}$, denoted by $Z_{\mathbb{R}}\left(F, P_{>}\right)$, is called a basic semi-algebraic set. It is well known that any semi-algebraic set is a finite union of basic semi-algebraic sets of $\mathbb{Q}[\mathbf{u}, \mathbf{y}]$. The set cs $:=\left\{(u, y) \in \mathbb{C}^{n} \mid f_{1}(u, y)=\right.$ $\left.0, \ldots, f_{s}(u, y)=0, p_{1}(u, y) \neq 0, \ldots, p_{r}(u, y) \neq 0\right\}$ is called the associated constructible set of $Z_{\mathbb{R}}\left(F, P_{>}\right)$.

In this section, we introduce the concept of the comprehensive triangular decomposition of a parametric basic semi-algebraic system and propose an algorithm to compute it.

Definition 11 Let $R:=[T, P]$ be a squarefree regular system of $\mathbb{Q}[\mathbf{u}, \mathbf{y}]$. We call the pair $A:=\left[T, P_{>}\right]$a squarefree semi-algebraic system (SFSAS). The system $R$ is called the associated regular system of $A$.

Definition 12 Let $\mathcal{S}$ be a basic semi-algebraic set of $\mathbb{Q}[\mathbf{u}, \mathbf{y}]$. Let cs be the associated constructible set of $\mathcal{S}$. A comprehensive triangular decomposition of $\mathcal{S}$ is a pair $\left(\mathcal{C},\left(\mathcal{A}_{C}, C \in \mathcal{C}\right)\right)$, where

- $\mathcal{C}$ is a finite partition of $\mathbb{R}^{d}$ into nonempty semi-algebraic sets,

- for each $C \in \mathcal{C}, \mathcal{A}_{C}$ is a finite set of SFSASes of $\mathbb{Q}[\mathbf{u}, \mathbf{y}]$ such that:

(i) either $\mathcal{A}_{C}$ is empty, which means that $\mathcal{S}(u)$ is empty for each $u \in C$;

(ii) or $\mathcal{A}_{C}=\{[\varnothing,\{\}]\}$, which implies that $\operatorname{cs}(u)$ is infinite for each $u \in C$;

(iii) or $C$ is a connected semi-algebraic set, each $A=\left[T, P_{>}\right] \in \mathcal{A}_{C}$ satisfies $\operatorname{mvar}(T)=\mathbf{y}$ and for each $u \in C$ we have:

- the associated regular systems of $\mathcal{A}_{C}$ specializes disjointly well at $u$, - for each $A \in \mathcal{A}_{C}, Z_{\mathbb{R}}(A(u))$ is not empty,

- $\mathcal{S}(u)=\cup_{A \in \mathcal{A}_{C}} Z_{\mathbb{R}}(A(u))$.

Next, we provide an algorithm for computing a CTD of a basic semi-algebraic set. It relies on a subroutine for decomposing real constructible sets into connected cylindrically arranged cells of $\mathbb{R}^{d}$. The subroutine can be easily described via the subroutines MPD, MakeCylindrical and MakeSemiAlgebraic in paper [8].

Calling sequence. $\mathrm{CAD}(\mathcal{C})$

Input. $\mathcal{C}:=\left\{C_{1}, \ldots, C_{e}\right\}$ is a set of pairwise disjoint constructible sets of $\mathbb{C}^{n}$ given by polynomials in $\mathbb{Q}[\mathbf{x}]$ such that $\mathbb{C}^{n}=\cup_{i=1}^{e} C_{i}$.

Output. A CAD $\mathcal{E}$ of $\mathbb{R}^{n}$ such that for each element $C$ of $\mathcal{C}$, the set $C \cap \mathbb{R}^{n}$ is a union of some cells in $\mathcal{E}$.

Step (1). For $1 \leq i \leq e$, apply operation MPD to the family of regular systems representing $C_{i}$, so as to obtain another family $\mathcal{R}_{i}$ of regular systems representing $C_{i}$ and whose zero sets are pairwise disjoint.

Step (2). Let $\mathcal{R}:=\cup_{i=1}^{e} \mathcal{R}_{i}$. Call algorithm MakeCylindrical $(\mathcal{R}, n)$, to compute a cylindrical decomposition $\mathcal{D}$ of $\mathbf{K}^{n}$ such that the zero set of each regular system in $\mathcal{R}$ is a union of some cells in $\mathcal{D}$. 
Step (3). Call algorithm MakeSemiAlgebraic to compute a CAD $\mathcal{E}$ of $\mathbb{R}^{n}$ such that, for each element $D$ of $\mathcal{D}$, the set $D \cap \mathbb{R}^{n}$ is a union of some cells in $\mathcal{E}$.

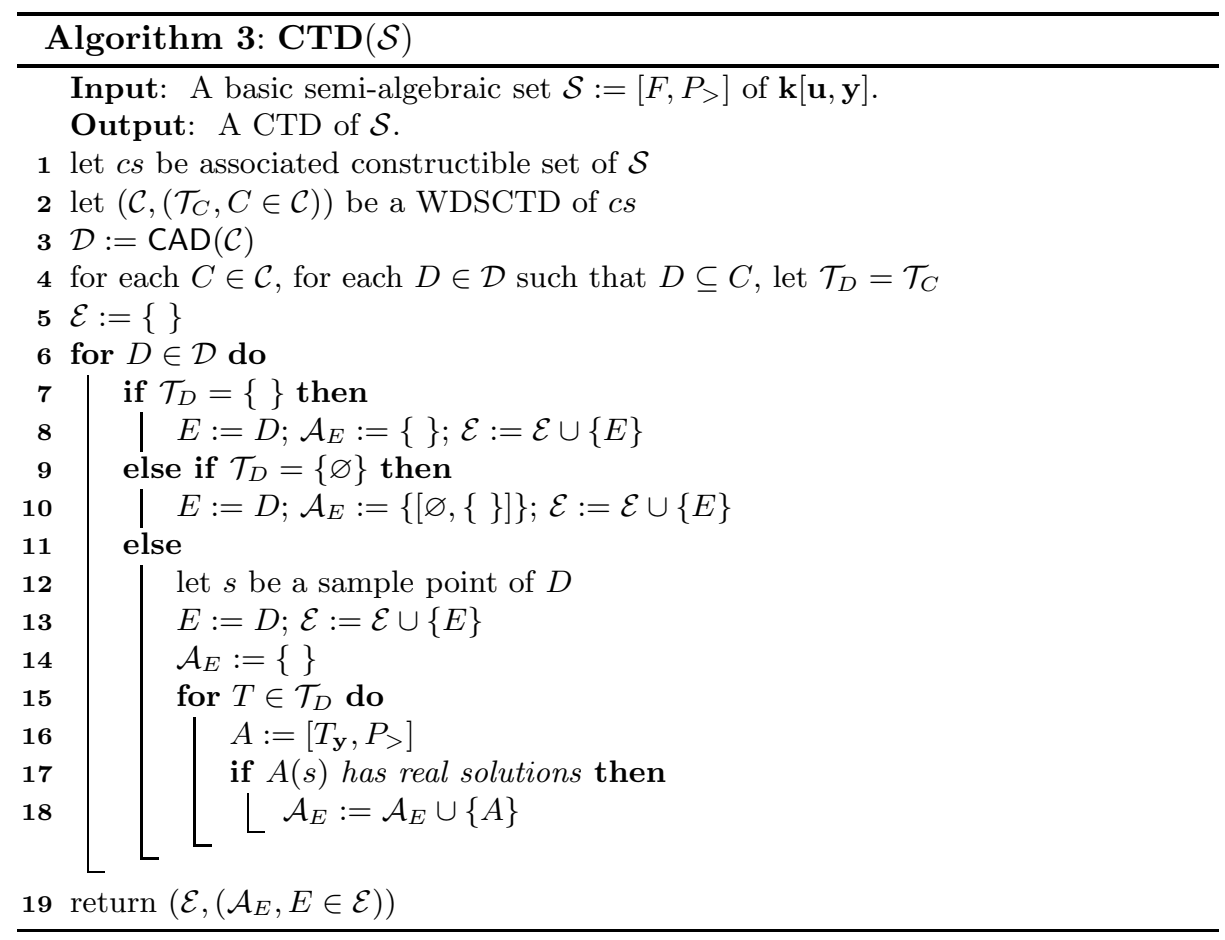

\section{Conclusion}

Based on the notion of a comprehensive triangular decomposition (CTD) presented in the last section, we have obtained a framework for analyzing the stability of the equilibria and compute the bifurcations of polynomial dynamical systems. Indeed, we can completely solve the problems introduced in Section 1.

Let us first have a look at Problem 1. Let $F(\mathbf{u}, \mathbf{x})$ be the right hand side polynomial equations of the dynamical system (1). It is usually required that $\mathbf{u}$ and $\mathbf{x}$ are both positive. Let $P(\mathbf{u}, \mathbf{x})$ be the corresponding set of positive inequality constraints. Let $\left(\mathcal{C},\left(\mathcal{A}_{C}, C \in \mathcal{C}\right)\right)$ be a CTD of $\mathcal{S}=\left[F, P_{>}\right]$. In the practice of dynamical systems, only the cells above which $\mathcal{S}$ has finitely many complex solutions are interesting. This fact has motivated our definition of the CTD of a semi-algebraic system. Let $C \in \mathcal{C}$ be a cell above which $\mathcal{S}$ has finitely many complex solutions, one of them at least being real, that is, a cell of type (iii) in Definition 12. The set $C$ is a connected semi-algebraic subsets of $\mathbb{R}^{d}$, above which $\mathcal{A}_{C}$ is a finite set of SFSASes whose solutions are disjoint graphs 
of continuous functions above $C$; moreover the union of the graphs of these functions is exactly $C \cap Z_{\mathbb{R}}(\mathcal{S})$. Therefore, Problem 1 is solved.

Next, we look at Problem 2. A first and direct approach consists of computing a CTD of the system $\mathcal{S}$ augmented with the inequalities $\Delta_{i}>0,1 \leq i \leq m$, where the $\Delta_{i}$ are the Hurwitz determinants, see Definition 1. A second approach consists of computing a CTD of the system $\mathcal{S}$ augmented with the inequality $\Delta_{m}>0$ only and then apply the Boundary Crossing Theorem, that is Theorem 13 .

Similarly, for each of the three other problems on bifurcation, we will first produce a semi-algebraic system by means of results in Section 2 and then apply CTD to solve it.

\section{References}

1. V.I. Arnold. Ordinary Differential Equations. Springer-Verlag, 1992.

2. C. Chen, J.H. Davenport, J. May, M. Moreno Maza, B. Xia, and R. Xiao. Triangular decomposition of semi-algebraic systems. In S.M. Watt, editor, Proceedings ISSAC 2010, pages 187-194, 2010.

3. J. Carr. Applications of Centre Manifold Theory. Springer-Verlag, 1981.

4. Jack Carr. Applications of Centre Manifold Theory. Springer-Verlag, New York, Heidelberg, Berlin, 1981.

5. C. Chen. Algebraic analysis of stability for biological systems and the implemetation of a software pakage (in chinese). Master's thesis, Peking University, 2006.

6. C. Chen, O. Golubitsky, F. Lemaire, M. Moreno Maza, and W. Pan. Comprehensive Triangular Decomposition, volume 4770 of Lecture Notes in Computer Science, pages 73-101. Springer Verlag, 2007.

7. C. Chen and M. Moreno Maza. Algorithms for computing triangular decompositions of polynomial systems. CoRR, abs/1104.0689, 2011.

8. C. Chen, M. Moreno Maza, B. Xia, and L. Yang. Computing cylindrical algebraic decomposition via triangular decomposition. In ISSAC'09, pages 95-102, 2009.

9. Guoting Chen and Jean Della Dora. Rational normal form for dynamical systems by Carleman linearization. In S. Dooley, editor, Proc. 1999 International Symposium on Symbolic and Algebraic Computation (ISSAC), pages 165-172, New York, 1999. ACM Press.

10. Guoting Chen and Jean Della Dora. An algorithm for computing a new normal form for dynamical systems. Journal of Symbolic Computation, 29(3):393-418, 2000 .

11. Guoting Chen, Jean Della Dora, and Laurent Stolovitch. Nilpotent normal form via Carleman linearization (for systems of ordinary differential equations). In S. Watt, editor, Proc. 1991 International Symposium on Symbolic and Algebraic Computation (ISSAC), pages 281-288, New York, 1991. ACM Press.

12. Frazer, R. A. and Duncan, W. J. On the criteria for the stability of small motions. Proceedings of the Royal Society of London. Series A, Containing Papers of a Mathematical and Physical Character, 124(795):642-654, jul 1929.

13. E. Freire, E. Gamero, E. Ponce, and L. García Franquelo. An algorithm for symbolic computation of center manifolds. In Proc. of ISAAC '88, pages 218-230, London, UK, 1989. Springer-Verlag.

14. A.T. Fuller. Conditions for a matrix to have only characteristic roots with negative real parts. Journal of Mathematical Analysis and Applications, 23:71-98, 1968. 
15. F.R. Gantmacher. The Theory of Matrices. Chelsea Publishing Company, New York, N.Y., 1959.

16. K. Gatermann, M. Eiswirtha, and A. Sensse. Toric ideals and graph theory to analyze Hopf bifurcations in mass action systems. Journal of Symbolic Computation, 40(6):1361-1382, 2005.

17. K. Gatermann and S. Hosten. Computational algebra for bifurcation theory. Journal of Symbolic Computation, 40(4-5):1180-1207, 2005.

18. L. Gonzalez, H. Lombardi, T. Recio, and M.-F. Roy. Sturm-habicht sequence. In ISSAC '89: Proceedings of the ACM-SIGSAM 1989 international symposium on Symbolic and algebraic computation, pages 136-146, New York, NY, USA, 1989. ACM.

19. John Guckenheimer, M. Myers, and B. Sturmfels. Computing Hopf bifurcations I. SIAM J. Num. Anal., 34(1):1-21, 1997.

20. John Guckenheimer, Mark Myers, and Bernd Sturmfels. Computing hopf bifurcations i. SIAM J. Numer. Anal., 34(1):1-21, 1997.

21. J. Hale and H. Koçak. Dynamics and Bifurcations. Springer-Verlag, New York, Berlin, Heidelberg, London, 1991.

22. Hoon Hong, Richard Liska, and Stanly Steinberg. Testing stability by quantifier elimination. Journal of Symbolic Computation, 24(2):161-187, 1997.

23. M'Hammed El Kahoui and Andreas Weber. Deciding hopf bifurcations by quantifier elimination in a software-component architecture. J. Symb. Comput., 30(2):161-179, 2000.

24. M'Hammed El Kahoui and Andreas Weber. Symbolic equilibrium point analysis in parameterized polynomial vector fields. In V. Ganzha, E. Mayr, and E. Vorozhtsov, editors, Computer Algebra in Scientific Computing (CASC 2002), pages 71-83, 2002.

25. Y.A. Kuznetsov. Elements of Applied Bifurcation Theory. Springer Verlag, 1998.

26. M. Laurent. Prion diseases and the "protein only" hypothesis: a theoretical dynamic study. Biochem. J., 318:35-39, 1996.

27. Xianping Liu, Robert M. Corless, and K. O. Geddes. Computation of center manifolds. Technical Report TR-00-15, at http://www.orcca.on.ca/TechReports, Ontario Research Centre for Computer Algebra, 2000. 12 pages.

28. R. K. Miller and A. N. Michel. Ordinary Differential Equations. Academic Press, 1982.

29. B. Mishra. Algorithmic Algebra. Springer-Verlag, New York, 1993.

30. A.H. Nayfeh. Method of Normal Forms. Wiley Series in Nonlinear Sciences. John Wiley \& Sons, New York, Chichester, Brisbane, 1993.

31. W. Niu and D. M. Wang. Algebraic approaches to stability analysis of biological systems. Mathematics in Computer Science, 1:507-539, 2008.

32. Lawrence Perko. Differential Equations and Dynamical Systems. Springer-Verlag New York, Inc., New York, NY, USA, 1991.

33. D.G. Schaeffer and M. Golubitsky. Singularities and Groups in Bifurcation Theory, volume 1. Springer-Verlag, New York, Berlin, Heidelberg, Tokyo, 1984.

34. L. Vallier. An algorithm for the computation of normal forms and invariant manifolds. In Proc. of ISSAC '93, pages 225-233, New York, NY, USA, 1993. ACM Press.

35. D.M. Wang and Z.M. Zheng. Differential Equations with Symbolic Computation. Birkhäuser Verlag, Basel, Boston, Berlin, 2005.

36. Dongming Wang and Bican Xia. Stability analysis of biological systems with real solution classfication. In M. Kauers, editor, Proc. 2005 International Symposium 
on Symbolic and Algebraic Computation (ISSAC), pages 354-361, New York, 2005. ACM Press.

37. Lu Yang. Recent advances on determining the number of real roots of parametric polynomials. J. Symb. Comput., 28(1-2):225-242, 1999.

38. Lu Yang, Xiaorong Hou, and Bican Xia. A complete algorithm for automated discovering of a class of inequality-type theorems. Science in China, Series $\mathbf{F}$, 44(6):33-49, 2001.

39. P. Yu and Y. Yuan. An efficient method for computing the simplest normal forms of vector fields. Int. J. Bifurcations \& Chaos, 13(1):19-46, 2003.

\section{A Example}

In this section we present a complete process for analyzing the stability of a biochemistry network by means of the tools presented in this paper.

\section{A.1 The description of the model}

In [26], Laurent proposed a model for the dynamics of diseases of the central nervous system caused by prions, such as scrapie in sheep and goat, and "mad cow disease" or Creutzfeldt-Jacob disease in humans. The model is based on the protein-only hypothesis, which assumes that infection can be spread by particular proteins (prions) that can exist in two isomeric forms. The normal form $\operatorname{Pr} P^{C}$ is harmless, while the infectious form $\operatorname{PrP}^{S_{C}}$ catalyzes a transformation from the normal form to itself. A natural question is whether a small amount of $\operatorname{Pr} P^{S_{C}}$ cause prion disease.

The generic kinetic scheme of prion diseases is illustrated as follows:

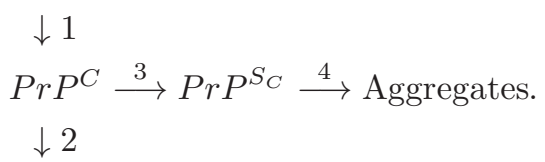

Denote by $\left[\operatorname{Pr} P^{C}\right]$ and $\left[\operatorname{Pr} P^{S_{C}}\right]$ be respectively the concentrations of $\operatorname{Pr} P^{C}$ and $\operatorname{Pr} P^{S_{C}}$. Let $\nu_{i}$ be the rate of Step $i$ for $i=1, \ldots, 4$. In the above diagram, Step 1 corresponds to the synthesis of native $\operatorname{Pr} P^{C}$, which is considered in the present analysis as a zero-order kinetic process, that is $\nu_{1}=k_{1}$ for some constant $k_{1}$. Output reactions (Steps 2 and 4 , which correspond to the degradation of native $\operatorname{Pr} P^{C}$ and to the formation of aggregates respectively) are taken as firstorder rate equations: $\nu_{2}=k_{2}\left[\operatorname{Pr} P^{C}\right], \nu_{4}=k_{4}\left[\operatorname{Pr} P^{S_{C}}\right]$. Step 3 corresponds to the transformation from $\operatorname{Pr} P^{C}$ to $\operatorname{PrP}^{S_{C}}$, which is a nonlinear process

$$
\nu_{3}=\left[\operatorname{Pr} P^{C}\right] \frac{a\left(1+b\left[\operatorname{Pr} P^{S_{C}}\right]^{n}\right)}{1+c\left[\operatorname{PrP}^{S_{C}}\right]^{n}} .
$$

Hence we can describe the model by the following differential equations:

$$
\begin{aligned}
& \frac{\mathrm{d}\left[P r P^{C}\right]}{\mathrm{d} t}=\nu_{1}-\nu_{2}-\nu_{3} \\
& \frac{\mathrm{d}\left[\operatorname{Pr} P^{S_{C}}\right]}{\mathrm{d} t}=\nu_{3}-\nu_{4}
\end{aligned}
$$


To simplify notation, we set $x=\left[\operatorname{Pr} P^{C}\right], y=\left[\operatorname{Pr} P^{S_{C}}\right]$. The model is therefore described by the dynamical system:

$$
\begin{aligned}
& \frac{\mathrm{d} x}{\mathrm{~d} t}=k_{1}-k_{2} x-a x \frac{\left(1+b y^{n}\right)}{1+c y^{n}} \\
& \frac{\mathrm{d} y}{\mathrm{~d} t}=a x \frac{\left(1+b y^{n}\right)}{1+c y^{n}}-k_{4} y
\end{aligned}
$$

where experiments suggest to set $b=2, c=1 / 20, n=4, a=1 / 10, k_{4}=50$ and $k_{1}=800$. Now we have:

$$
\left\{\begin{array} { l } 
{ \frac { \mathrm { d } x } { \mathrm { d } t } = f _ { 1 } } \\
{ \frac { \mathrm { d } y } { \mathrm { d } t } = f _ { 2 } }
\end{array} \text { with } \left\{\begin{array}{l}
f_{1}=\frac{16000+800 y^{4}-20 k_{2} x-k_{2} x y^{4}-2 x-4 x y^{4}}{20+y^{4}} \\
f_{2}=\frac{2\left(x+2 x y^{4}-500 y-25 y^{5}\right)}{20+y^{4}}
\end{array} .\right.\right.
$$

Recall that a constant solution of the above differential equations is called an equilibrium, that is a point $(x, y) \in \mathbb{R}^{2}$ at which the right hand side equations vanish. By Routh-Hurwitz criterion $(x, y)$ is asymptotically stable if

$$
\Delta_{1}=-\left(\frac{\partial f_{1}}{\partial x}+\frac{\partial f_{2}}{\partial y}\right)>0 \text { and } a_{2}=\frac{\partial f_{1}}{\partial x} \cdot \frac{\partial f_{2}}{\partial y}-\frac{\partial f_{1}}{\partial y} \cdot \frac{\partial f_{2}}{\partial x}>0 .
$$

In system (6), let $p_{1}$ and $p_{2}$ be respectively the numerators of $f_{1}$ and $f_{2}$. The parametric semi-algebraic sets $\mathcal{S}_{1}:\left\{p_{1}=p_{2}=0, k_{2}>0\right\}$ and $\mathcal{S}_{2}:\left\{p_{1}=\right.$ $\left.p_{2}=0, k_{2}>0, \Delta_{1}>0, a_{2}>0\right\}$ encode respectively the equilibria and the asymptotically stable hyperbolic equilibria of System (6).

\section{A.2 Studying the equilibria with CTD}

Firstly, we compute a CTD of $\mathcal{S}_{1}$. Let

$$
\begin{aligned}
R_{1} & =100000 k_{2}^{8}+1250000 k_{2}^{7}+5410000 k_{2}^{6}+8921000 k_{2}^{5}-9161219950 k_{2}^{4} \\
& -5038824999 k_{2}^{3}-1665203348 k_{2}^{2}-882897744 k_{2}+1099528405056 .
\end{aligned}
$$

The polynomial $R_{1}$ has four real roots, two of them are positive. We denote them by $0<\alpha_{1}<\alpha_{2}$. Then the real line $\mathbb{R}$ is partitioned into 6 connected cells: $k_{2} \leq 0,0<k_{2}<\alpha_{1}, k_{2}=\alpha_{1}, \alpha_{1}<k_{2}<\alpha_{2}, k_{2}=\alpha_{2}$ and $k_{2}>\alpha_{2}$. For the first cell, namely $k_{2} \leq 0$, there is no associated SFSAS, which implies that $\mathcal{S}$ has no real solutions. The second, fourth and sixth cells are associated with the same SFSAS, which is

$$
A_{1}:=\left\{\begin{aligned}
\left(2 y^{4}+1\right) x-25 y^{5}-500 y & =0 \\
\left(k_{2}+4\right) y^{5}-64 y^{4}+\left(2+20 k_{2}\right) y-32 & =0 \\
k_{2} & >0 .
\end{aligned}\right.
$$

The third and fifth cells are associated with the SFSAS $A_{2}$, which will not be displayed here due to its size. For each of the sixth cells, we can compute a sample point and substitute it into the corresponding SFSAS. Then we obtain the number of real solutions above the six cells, which are respectively $0,1,2,3,2,1$. To summarize, we have the following conclusion. 
Conclusion 1 Assume $k_{2}>0$. If $R_{1}>0$, then System (6) has 1 equilibrium; if $R_{1}=0$, then System (6) has 2 equilibria; if $R_{2}<0$, then System (6) has 3 equilibria.

Similarly, we can also compute a CTD of $\mathcal{S}_{2}$ and then count the number of asymptotically stable hyperbolic equilibria above each cell. Let $R_{2}$ be the following polynomial.

$$
\begin{aligned}
R_{2} & =10004737927168 k_{2}^{9}+624166300700672 k_{2}^{8}+7000539052537600 k_{2}^{7} \\
& +45135589467012800 k_{2}^{6}-840351411856453750 k_{2}^{5}-50098004352248446875 k_{2}^{4} \\
& -27388168989455000000 k_{2}^{3}-8675209266696000000 k_{2}^{2} \\
& +102960917356800000000 k_{2}+5932546064102400000000 .
\end{aligned}
$$

The following conclusion summarizes the conditions for the stability and bifurcation of System (6).

Conclusion 2 Assume $k_{2}>0$. If $R_{1}>0$ (Figures 1 and 3 ), then the system has one hyperbolic equilibrium, which is asymptotically stable; if $R_{1}<0$ and $R_{2} \neq 0$ (Figure 2), then the system has three hyperbolic equilibria, two of which are asymptotically stable, the other one being unstable; if $R_{1}=0$ or $R_{2}=0$ hold, the system experiences a bifurcation.

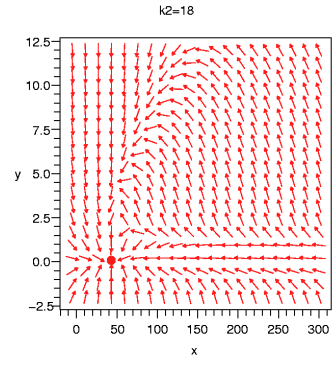

Figure 1

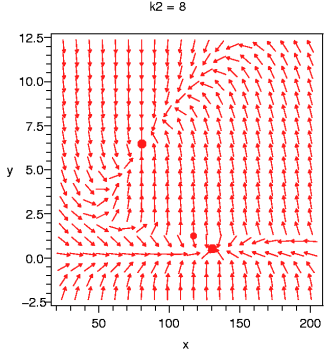

Figure 2

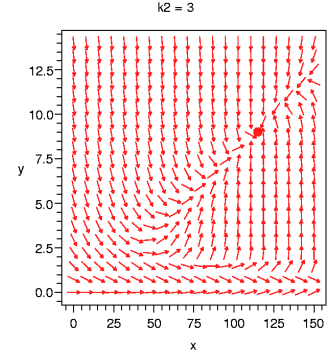

Figure 3

Remark 5 This generalizes the illustrated results of Fig.1(c) in [26], where only concrete values of $k_{2}$ are given to make sure that system (6) is bistable. By symbolic methods presented here, we can give the precise condition.

\section{A.3 Explanation of the experimental results}

From these figures, we also observe that, in Figure 1, the concentration of $\operatorname{Pr} P^{S_{C}}$ ( $y$-coordinate) finally becomes low and thus the system enters a harmless state. Conversely, in Figure 3 the concentration of $\operatorname{Pr} P^{S_{C}}$ goes high and thus the systems enters a pathogenic state. In Figure 2, the system exhibits bistability, the initial concentrations of $\operatorname{PrP}^{S_{C}}$ determines whether the final state pathogenic or not. We thus deduce the following facts, as stated in paper [26]: 
- The turnover rate $k_{2}$ determines whether it is possible for a pathogenic state to occur.

- As an answer to our question, a small amount of $\operatorname{Pr}^{S_{C}}$ does not lead to a pathogenic state when $k_{2}$ is large enough.

- Compounds that inhibit addition of $\operatorname{Pr} P^{S_{C}}$ can be seen as a possible therapy against prion diseases. However, compounds that increase the turnover rate $k_{2}$ would be the best therapeutic strategy against prion diseases. 